\title{
Generalised zonal modes in stationary axisymmetric plasmas
}

\author{
J. P. Graves ${ }^{1}$, C. Wahlberg ${ }^{2}$ \\ 1 École Polytechnique Fédérale de Lausanne (EPFL), \\ Swiss Plasma Center (SPC), CH-1015 Lausanne, Switzerland and \\ 2 Department of Astronomy and Space Physics, P.O. Box 515, Uppsala University, SE-751 20 Uppsala, Sweden
}

(Dated: November 3, 2016)

The MHD model enables derivation and analysis of the rich structure of geodesic acoustic modes (GAMs) and zonal modes in axisymmetric magnetic confined plasmas. The modes are identifiable from a single dispersion relation as two branches of slow magnetosonic continua. The lower frequency branch can be identified as a zonal flow, which in the simplified limit of static plasmas, has vanishing magnetic component. It is shown in this contribution that axisymmetric, and lesser known nonaxisymmetric, zonal modes can be derived from MHD and kinetic models. The work provides a comprehensive derivation of the GAMs and zonal flow continua in static and stationary toroidally rotating plasmas, and investigates the exact solution and structure of a generalised family of zonal modes.

\section{INTRODUCTION}

Geodesic modes (GAMs) and zonal flows (ZFs) are well known to be connected to the transport properties of confined plasmas $[1-3]$. Both modes are usually considered to be axisymmetric in axisymmetric confined plasmas. It is perhaps less well known that both modes are two roots of a single dispersion that can be derived from an ideal MHD model [4]. Importantly, the ideal MHD model provides a means of calculating the electromagnetic corrections of these dominantly electrostatic continuum modes, and reveal the global characteristics of the modes. While standard gyrokinetic models struggle with these features, the MHD results of Ref. [5] appear to agree with experimental measurements of GAMs using magnetic probes. Also, perhaps little known is the effect that equilibrium toroidal plasma rotation (e.g. generated by momentum input from neutral beam injection) has on GAMs and ZFs. In addition to enriching the magnetic spectrum and global structure of the modes, toroidal rotation modifies their oscillation frequencies, despite the fact that the modes ordinarily have zero toroidal mode number, and are hence not Doppler shifted by a factor $n \Omega$ when viewing in the laboratory frame (here $n$ is the toroidal mode number, and $\Omega$ is the toroidal rotation frequency). This is of particular importance for zonal flows, which in the absence of toroidal rotation, have zero frequency, and thus would be undetectable by magnetic probes if the toroidal plasma rotation were zero. In addition to theoretical identification [4] of low frequency $n=0$ zonal modes in rotating plasmas, experiments at JET frequently observe low frequency $n=0$ modes near the transition between L-mode and $\mathrm{H}$-mode (see e.g. the $n=0$ mode of Figure 1 in Ref. [6]). These modes, which do not scale with the sound frequency, are colloquially known as M-modes [7] because of their (M)agnetic characteristics.

The inclusion of toroidal plasma rotation in stability modelling illustrates that in addition to connections be- tween GAMs, zonal flows and turbulence, such continuum modes also interact with MHD instabilities. In Ref. [8] it was shown that continuum modes oscillating at the Brunt-Väisälä [9] frequency directly impacts the stabilily threshold of the internal kink mode, and that inconsistent treatment of the effect of toroidal rotation on the continuum mode would lead to erroneous results. The mode oscillating at the Brunt-Väisälä frequency is in fact a finite $n$ zonal mode, which arises as one of two roots [10] from a dispersion relation that also defines the geodesic acoustic mode. In Ref. [5] the electromagnetic global structure of these two modes was allucidated, both for cases of $n=0$ and for non-axisymmetric modes. In the limit of vanishing toroidal rotation the zonal mode coincides with the zero frequency non-axisymmetric modes identified by Goedbloed in Ref. [11]. Similar modes in rotating plasmas have recently [12] been identified numerically using the FINESSE [13] code. In the current paper we present an instructive derivation of finite $n$ GAMs and ZF continua in the presence of toroidal rotation. After that we focus on the low frequency zonal flow mode for $n=0$ and finite $n$, and show that its mode structure can be obtained exactly in the limit of vanishing toroidal rotation using an ideal MHD model and with a drift kinetic-MHD model. In addition to the academic interest of exact finite $n$ zonal flows, the work provides an important step towards identifying the interaction between continuum modes and kinetic instabilities, particularly in the presence of equilibrium plasma rotation.

The paper is organised as follows. In Section II we derive zonal flow and GAM mode continua in toroidal rotating plasmas in the electrostatic limit of the MHD model. A brief kinetic description of equilibria with toroidal rotation is also outlined, and it is shown that the parallel component of the momentum equation of ideal MHD does not have to be imposed. Section III contains an exact treatment of zonal flows for the MHD model with vanishing toroidal rotation. In addition section III is dedicated to illustrating the mode structure, and it is 
shown that the concept of convective cells associated with $n=0$ zonal flows also follows for non-axisymmetric zonal flows. In section IV it is shown that the results of Section III are reproduced using a fully electromagnetic drift kinetic-MHD model. Section V is dedicated to summarising what has been shown in the paper, for a discussion on precisely how non-axisymmetric zonal flows couple to instabilities, and briefly the next steps to be taken in describing kinetic-MHD instabilities in rotating plasmas.

\section{MHD MODEL OF CONTINUUM MODES IN TOROIDALLY ROTATING PLASMAS}

In this section we review plasma equilibria with toroidal rotation assuming an isothermal description of the plasma, and then derive GAM and zonal flow continua in a rotating plasma. A kinetic derivation of the equilibrium is an important first step for a kineticMHD study into zonal flows, since the magnetic equilibrium equations must be shown to be compatible with the linearly perturbed equations that describe kinetic MHD instabilities and continua. A particular feature of kinetic-MHD is that a momentum equation parallel to the magnetic field is not required, so the below equilibrium derivation starts from kinetic theory, and thus does not impose from the start the parallel momentum equation of ideal MHD.

\section{A. MHD rotating equilibria}

An axisymmetric equilibrium field of the form

$$
\boldsymbol{B}=F(\psi) \nabla \phi+\nabla \phi \times \nabla \psi
$$

is assumed where $\phi$ is the toroidal angle, $\psi$ the poloidal flux and $F$ is a flux function. A kinetic treatment in strongly $\boldsymbol{E} \times \boldsymbol{B}$ flowing plasmas in all collisionality regimes has shown [14] that the distribution of ions or electrons (generically species $j$ ) conform to a Maxwellian

$$
F_{j}=\frac{\bar{n}_{j}(\psi)}{\left(2 \pi T_{j}(\psi)\right)^{3 / 2}} \exp \left(-\frac{\mathcal{E}_{\text {rot }}}{T_{j}(\psi)}\right) .
$$

in modified energy,

$$
\mathcal{E}_{\text {rot }}=\frac{m_{j} w_{\|}^{2}}{2}+\mu B-\frac{m_{j} \Omega^{2} R^{2}}{2}+q_{j} \Phi_{1},
$$

where $w_{\|}$is the parallel fluctuation velocity, and $\mu=$ $m_{j} w_{\perp}^{2} /(2 B)$ is the magnetic moment. As will be seen the density and pressure distributions of the ions and electrons are not flux functions, but the distributions are isothermal, i.e. $T_{j}=T_{j}(\psi)$. The electric field that occurs in the equilibria of such plasmas are expanded in orders of larmor radius

$$
\boldsymbol{E}=-\nabla \Phi_{0}-\nabla \Phi_{1}
$$

and it is found that the perpendicular flow velocity $\boldsymbol{U}_{\perp}$ is related to the leading order electric field $\boldsymbol{E}_{0}=-\boldsymbol{\nabla} \Phi_{0}$ via Ohm's law $\boldsymbol{E}_{0}+\boldsymbol{U}_{\perp} \times \boldsymbol{B}=0$ while the leading order parallel flow vector is identified by the condition that the leading order flow is purely toroidal, specifically

$$
\boldsymbol{U}=R^{2} \Omega(\psi) \nabla \phi \text { with } \Omega=-\frac{d \Phi_{0}}{d \psi} .
$$

The purely toroidal flow of Eq. (3) prevents magnetic pumping, a feature that arises if the flow moves in a direction of varying magnetic field strength. It is clearly seen that Eq. (3) readily obeys Ohm's law, incompressibility at equilibrium $\boldsymbol{\nabla} \cdot \boldsymbol{U}=0$, and the continuity equation.

The fact that $\mathcal{E}_{\text {rot }}$ depends on the centrifugal effect $\Omega^{2} R^{2}$ indicates that moments of the distributions of ions will not be flux functions (due to $R$ not being a flux function) when $\Omega \neq 0$. In contrast, due to their low mass, electrons would not significantly feel the centrifugal contribution $-m_{j} \Omega^{2} R^{2} / 2$ in $\mathcal{E}_{\text {rot }}$. However, the quasineutrality assumption of the plasma would require that ions and electrons experience the same displacement due to finite $\Omega$. This apparent anomaly is solved by consideration of a higher order (in Larmor radius) electric potential $\Phi_{1}$. This is undertaken by first noting that the density function $\bar{n}_{j}$ is not the particle density, while the density itself is obtained by integration over velocity

$$
\begin{aligned}
& n_{j}=2 \pi \int_{-\infty}^{\infty} d w_{\|} \int_{0}^{\infty} d w_{\perp} w_{\perp} \frac{\bar{n}_{j}(\psi)}{\left(2 \pi T_{j}(\psi)\right)^{3 / 2}} \\
& \quad \exp \left[-\frac{1}{T_{j}(\psi)}\left(\frac{m_{j} w_{\|}^{2}}{2}+\frac{m_{j} w_{\perp}^{2}}{2}-\frac{m_{j} \Omega^{2} R^{2}}{2}+q_{j} \Phi_{1}\right)\right] .
\end{aligned}
$$

This gives:

$$
n_{j}(\psi, \theta)=\bar{n}_{j}(\psi) \exp \left(\frac{m_{j} \Omega^{2} R^{2}}{2 T}-\frac{q_{j} \Phi_{1}}{T_{j}}\right) .
$$

We will see that $\Phi_{1}$ will reduce the outward shift of ions, and increase the outward shift of electrons in order that the plasma is shifted in unison. Note also that the pressure for each species can easily be constructed

$$
\begin{aligned}
P_{j} & =\int d w^{3} F_{j} \frac{1}{2}\left(w_{\|}^{2}+\frac{w_{\perp}^{2}}{2}\right) \\
& =\exp \left(\frac{m_{j} \Omega^{2} R^{2}}{2 T_{j}}-\frac{q_{j} \Phi_{1}}{T_{j}}\right) \times \\
& \int d w^{3} \frac{1}{2}\left(w_{\|}^{2}+\frac{w_{\perp}^{2}}{2}\right) \frac{\bar{n}_{j}(\psi)}{\left(2 \pi T_{j}(\psi)\right)^{3 / 2}} \exp \left(-\frac{\mathcal{E}}{T_{j}(\psi)}\right)
\end{aligned}
$$

This clearly gives

$$
\begin{aligned}
P_{j}(\psi, \theta) & =\bar{n}_{j}(\psi) T_{j}(\psi) \exp \left(\frac{m_{j} \Omega^{2} R^{2}}{2 T_{j}}-\frac{q_{j} \Phi_{1}}{T_{j}}\right) \\
& =n_{j}(\psi, \theta) T_{j}(\psi),
\end{aligned}
$$

where the poloidal dependence enters via $R(\psi, \theta)$, the poloidal angle $\theta$ being arbitrary at this point. The only 
way in which electrons can be brought into line with the ion centrifugal effect is with the potential

$$
\Phi_{1}(\psi, \theta)=\bar{\Phi}_{1}(\psi)\left(\frac{R}{R_{0}}\right)^{2}
$$

We now use quasi-neutrality equation $\sum_{j} q_{j} n_{j}=0$ to find $\bar{\Phi}_{1}$. If we neglect hot ions, and let $q_{i}=e$ and $q_{e}=-e$ then quasi-neutrality $(\mathrm{QN})$ is solved trivially and exactly by $n_{i}=n_{e}$, which requires that

$$
\exp \left(\frac{m_{i} \Omega^{2} R^{2}}{2 T_{i}}-\frac{e \Phi_{1}}{T_{i}}\right)=\exp \left(\frac{m_{e} \Omega^{2} R^{2}}{2 T_{e}}+\frac{e \Phi_{1}}{T_{e}}\right),
$$

or

$$
\frac{m_{i} \Omega^{2} R^{2}}{2 T_{i}}-\frac{e \bar{\Phi}_{1} R^{2}}{T_{i} R_{0}^{2}}=\frac{m_{e} \Omega^{2} R^{2}}{2 T_{e}}+\frac{e \bar{\Phi}_{1} R^{2}}{T_{e} R_{0}^{2}} .
$$

The electron mass can be ignored, and upon simple rearrangement we find,

$$
\bar{\Phi}_{1}=\frac{m_{i} \Omega^{2} R_{0}^{2}}{2 e} \frac{T_{e}}{T_{i}+T_{e}}
$$

which on substituting into either the ion or electron densities (or pressures) we have

$$
\begin{aligned}
& n_{i, e}=\bar{n} \exp \left(\frac{m_{i} \Omega^{2} R^{2}}{2\left(T_{e}+T_{i}\right)}\right) \\
& P_{i, e}=\bar{n} T_{i, e} \exp \left(\frac{m_{i} \Omega^{2} R^{2}}{2\left(T_{e}+T_{i}\right)}\right)
\end{aligned}
$$

where we again we recall that $n_{i}=n_{e}=n$ has already been assumed. From the above it is seen that for $T_{i}=T_{e}$, the centrifugal shift of the ions has been halved by the electrostatic effect, and the net radial shift of the electrons meets that of the ions. In order to explicitly obtain $\Phi_{1}$ for the more general case where we have arbitrary bulk ion charge $q_{i}=Z_{i} e$ and also hot ions included with charge $q_{h}=Z_{h} e$ we have to expand the exponentials assuming small argument. This clearly gives (on dropping the electron mass) the QN equation $\left(n_{i} Z_{i}+n_{h} Z_{h}-n_{e}=0\right)$ :

$$
\begin{gathered}
n_{i} Z_{i}\left(\frac{m_{i} \Omega^{2} R^{2}}{2 T_{i}}-\frac{Z_{i} e \bar{\Phi}_{0} R^{2}}{T_{i} R_{0}^{2}}\right)+n_{e} \frac{e \bar{\Phi}_{1} R^{2}}{T_{e} R_{0}^{2}}+ \\
n_{h} Z_{h}\left(\frac{m_{h} \Omega^{2} R^{2}}{2 T_{h}}-\frac{Z_{h} e \bar{\Phi}_{1} R^{2}}{T_{h} R_{0}^{2}}\right)=0,
\end{gathered}
$$

which simply yields,

$$
\bar{\Phi}_{1}=\frac{R_{0}^{2} \Omega^{2}}{2 e}\left\{\frac{\frac{n_{h} Z_{h} m_{h}}{T_{h}}+\frac{n_{i} Z_{i} m_{i}}{T_{i}}}{\frac{n_{e}}{T_{e}}+\frac{Z_{i} n_{i}}{T_{i}}+\frac{n_{h} Z_{h}}{T_{h}}}\right\} .
$$

Let us now consider the perpendicular momentum equation, which is universally valid for microscopic and macroscopic flows that are dominantly due to $\boldsymbol{E} \times \boldsymbol{B}$ motion [15]:

$$
\left.\rho \frac{d \boldsymbol{U}}{d t}\right|_{\perp}=\boldsymbol{J} \times \boldsymbol{B}+\left[\left(P_{\perp}-P_{\|}\right) \boldsymbol{\kappa}-\nabla_{\perp} P_{\perp}\right]
$$

where $\rho=\sum_{j} \rho_{j}$ and $P=\sum_{j} P_{j}$, and

$$
\frac{d}{d t}=\frac{\partial}{\partial t}+\boldsymbol{U} \cdot \nabla
$$

which in the isotropic pressure limit conforms to the perpendicular components of the standard momentum equation of ideal MHD equation. In contrast, the parallel component of the ideal MHD momentum equation is not always reliable, though for many studies the parallel component of the momentum equation is either not required, or is substituted by a kinetic treatment. The lack of use of the parallel momentum equation for linear kineticMHD problems is described in Section IV. Despite the obvious existence of parallel electric fields arising from $\Phi_{1}$, it is shown below that the parallel analogue of Eq. (5) is valid for the strongly rotating isotropic equilibrium problem, and that qausi-neutrality is accounted for exactly for multiple species of ions and electrons. As well as verifying a solid base for kinetic-MHD stability/continua problems, the remainder of this subsection also serves to identify properties and definitions that will be relied on later.

From

$$
\begin{aligned}
\boldsymbol{\nabla} P & =\left(\boldsymbol{\nabla} \psi \frac{\partial}{\partial \psi}+\nabla \theta \frac{\partial}{\partial \theta}+\nabla \phi \frac{\partial}{\partial \phi}\right) P, \\
\boldsymbol{B} & =F \boldsymbol{\nabla} \phi+\nabla \phi \times \nabla \psi
\end{aligned}
$$

and using the definition for the Jacobian $\mathcal{J}$

$$
\mathcal{J}^{-1}=\nabla \psi \cdot(\nabla \theta \times \nabla \phi)
$$

we obtain

$$
\boldsymbol{b} \cdot \nabla P=\frac{1}{\mathcal{J} B}\left(\frac{F \mathcal{J}}{R^{2}} \frac{\partial}{\partial \phi}+\frac{\partial}{\partial \theta}\right) P
$$

where $\boldsymbol{b}=\boldsymbol{B} / B$ and $\partial P / \partial \phi=0$ due to axisymmetry.

Consider the total pressure that we have derived in the kinetic analysis:

$$
P=\sum_{j} \bar{n}_{j} T_{j} \exp \left(\frac{m_{j} \Omega^{2} R^{2}}{2 T_{j}}-\frac{q_{j} \bar{\Phi}_{1} R^{2}}{T_{j} R_{0}^{2}}\right) .
$$

Since $\partial R / \partial \phi=0$, we have,

$$
\begin{aligned}
\boldsymbol{b} \cdot \nabla P & =\frac{1}{\mathcal{J} B} \sum_{j} \bar{n}_{j} T_{j} \frac{\partial}{\partial \theta} \exp \left(\frac{m_{j} \Omega^{2} R^{2}}{2 T_{j}}-\frac{q_{j} \bar{\Phi}_{1} R^{2}}{T_{j} R_{0}^{2}}\right) \\
& =\frac{1}{\mathcal{J} B} \sum_{j} P_{j}(\psi, \theta)\left(\frac{m_{j} \Omega^{2}}{2 T_{j}}-\frac{q_{j} \bar{\Phi}_{1}}{T_{j} R_{0}^{2}}\right) \frac{\partial R^{2}}{\partial \theta} .
\end{aligned}
$$


Employing $n_{j}=P_{j} / T_{j}$, and $\rho_{j}=m_{j} n_{j}$ it follows that

$$
\boldsymbol{b} \cdot \boldsymbol{\nabla} P=\frac{R}{\mathcal{J} B} \frac{\partial R}{\partial \theta}\left(\Omega^{2} \sum_{j} \rho_{j}-\frac{2 \bar{\Phi}_{1}}{R_{0}^{2}} \sum_{j} q_{j} n_{j}\right) .
$$

Now, from QN we have $\sum_{j} q_{j} n_{j}=0$ so that the term involving $\Phi_{1}$ in $\boldsymbol{b} \cdot \nabla P$ cancels. Also, since $\rho=\sum_{j} \rho_{j}$ we finally have

$$
\boldsymbol{b} \cdot \nabla P=\rho \frac{R \Omega^{2}}{\mathcal{J} B} \frac{\partial R}{\partial \theta} .
$$

It remains to calculate the parallel component of $\rho(\boldsymbol{U}$. $\nabla) U$,

$$
\boldsymbol{b} \cdot\{\rho(\boldsymbol{U} \cdot \boldsymbol{\nabla}) \boldsymbol{U}\}=\rho \frac{1}{R} \boldsymbol{b} \cdot\left\{U \frac{\partial}{\partial \phi}\left(U \boldsymbol{e}_{\phi}\right)\right\},
$$

where $\boldsymbol{e}_{\phi}$ is the unit vector in the toroidal direction. Defining the unit vector in the (major) radial direction

$$
\boldsymbol{e}_{R} \equiv \frac{\nabla R}{|\nabla R|}=-\frac{d \boldsymbol{e}_{\phi}}{d \phi}
$$

we then have the inertia that balances the centrifugal force due to toroidal rotation $(\boldsymbol{U} \cdot \boldsymbol{\nabla}) \boldsymbol{U}=-\Omega^{2} R \boldsymbol{e}_{R}$. Thus,

$$
\boldsymbol{b} \cdot\{\rho(\boldsymbol{U} \cdot \boldsymbol{\nabla}) \boldsymbol{U}\}=-\rho R \Omega^{2} \boldsymbol{b} \cdot \boldsymbol{\nabla} R
$$

where we have used $|\nabla R|=1$. Now, using

$$
\boldsymbol{\nabla} R=\frac{\partial R}{\partial \theta} \boldsymbol{\nabla} \theta+\frac{\partial R}{\partial \psi} \boldsymbol{\nabla} \psi \text { and } \boldsymbol{b}=\frac{F \boldsymbol{\nabla} \phi+\boldsymbol{\nabla} \phi \times \nabla \psi}{B}
$$

together with the definition of the Jacobian (6) we have

$$
\boldsymbol{b} \cdot \boldsymbol{\nabla} R=\frac{1}{\mathcal{J} B} \frac{\partial R}{\partial \theta}
$$

so that finally we have

$$
\boldsymbol{b} \cdot\{\rho(\boldsymbol{U} \cdot \boldsymbol{\nabla}) \boldsymbol{U}\}=-\rho \frac{R \Omega^{2}}{\mathcal{J} B} \frac{\partial R}{\partial \theta} .
$$

This is a useful result in itself, however, we can equate Eqs. (9) and (8) and thus obtain from the kinetic treatment presented in this section:

$$
\boldsymbol{b} \cdot\{\rho(\boldsymbol{U} \cdot \boldsymbol{\nabla}) \boldsymbol{U}\}=-\boldsymbol{b} \cdot \boldsymbol{\nabla} P .
$$

This equation describes parallel force balance and we note that the Larmor radius correction to the electric field, i.e. on $\Phi_{1}$ does not appear explicitly in the equation, but its effect is crucial. To conclude, in the isotropic pressure limit, the kinetic treatment of strongly rotating plasma equilibria overviewed here is consistent with the full ideal MHD momentum equation at equilibrium,

$$
\rho(\boldsymbol{U} \cdot \nabla) \boldsymbol{U}=\boldsymbol{J} \times \boldsymbol{B}-\nabla P .
$$

From this robust basis we are ready to investigate an ideal MHD description of GAMs and zonal flows in rotating plasmas, and a kinetic treatment later on.

\section{B. GAMs and Zonal flows in isothermal ideal MHD rotating plasmas}

For a description of linear perturbed ideal MHD in a rotating plasma we follow the approach by Frieman and Rotenburg [16]. In this work, a Lagrangian displacement $\boldsymbol{\xi}$ is employed, which can be defined in terms of the Lagrangian perturbed fluid velocity as

$$
\boldsymbol{\Delta} \boldsymbol{U} \equiv \frac{d \boldsymbol{\xi}}{d t}=\frac{\partial \boldsymbol{\xi}}{\partial t}+\boldsymbol{U} \cdot \boldsymbol{\nabla} \boldsymbol{\xi}
$$

where the perturbed Lagrangian flow $\boldsymbol{\Delta} \boldsymbol{U}$ is related to the Eulerian perturbed vectors $\boldsymbol{X}$ (including the fluid velocity) as $\boldsymbol{\Delta} \boldsymbol{X}=\boldsymbol{\delta} \boldsymbol{X}+\boldsymbol{\xi} \cdot \boldsymbol{\nabla} \boldsymbol{X}$ so that Ohm's law

$$
\delta E_{\perp}=-U \times \delta B-\delta U \times B
$$

can be written in terms of $\boldsymbol{\xi}$ via the Eulerian perturbed fluid velocity

$$
\boldsymbol{\delta} \boldsymbol{U}=\frac{\partial \boldsymbol{\xi}}{\partial t}+\boldsymbol{U} \cdot \boldsymbol{\nabla} \boldsymbol{\xi}-\boldsymbol{\xi} \cdot \boldsymbol{\nabla} \boldsymbol{U} .
$$

Using this definition of the displacement allows the ideal MHD equations of so called stationary plasmas to be identical to those of ideal linear perturbed MHD equations of static plasmas, except for a change to the momentum equation:

$$
\begin{gathered}
\rho\left[\frac{\partial^{2} \boldsymbol{\xi}}{\partial t^{2}}+2(\boldsymbol{U} \cdot \nabla) \frac{\partial \boldsymbol{\xi}}{\partial t}+(\boldsymbol{U} \cdot \nabla)[(\boldsymbol{U} \cdot \nabla) \boldsymbol{\xi}]\right]- \\
\boldsymbol{\nabla} \cdot[\rho \boldsymbol{\xi}(\boldsymbol{U} \cdot \boldsymbol{\nabla}) \boldsymbol{U}]=\boldsymbol{\delta} \boldsymbol{J} \times \boldsymbol{B}+\boldsymbol{J} \times \boldsymbol{\delta} \boldsymbol{B}-\boldsymbol{\nabla} \delta P
\end{gathered}
$$

where

$$
\boldsymbol{\delta} \boldsymbol{B}=\boldsymbol{\nabla} \times(\boldsymbol{\xi} \times \boldsymbol{B})=\boldsymbol{\nabla} \times\left(\boldsymbol{\xi}_{\perp} \times \boldsymbol{B}\right)
$$

and thus from Amperes law

$$
\boldsymbol{\delta} \boldsymbol{J}=\boldsymbol{\nabla} \times \boldsymbol{\delta} \boldsymbol{B}=\boldsymbol{\nabla} \times\left[\boldsymbol{\nabla} \times\left(\boldsymbol{\xi}_{\perp} \times \boldsymbol{B}\right)\right],
$$

and the full perturbed pressure follows from ideal MHD's equation of state:

$$
\delta P=-\boldsymbol{\xi} \cdot \nabla P-\Gamma P \nabla \cdot \boldsymbol{\xi}
$$

with $\Gamma$ the adiabatic index.

The advantages of using the Frieman and Rotenburg [16] for mode analysis are tremendous, especially for an investigation of electrostatic modes, where from the start specific forms of the displacement $\boldsymbol{\xi}$ can be chosen so that $\boldsymbol{\delta} \boldsymbol{J}=0$ and $\boldsymbol{\delta} \boldsymbol{B}=0$. In particular, it will be shown below that GAMs and zonal flow continua in rotating plasmas can be derived intuitively, rigourously and briefly. First some identities are required. We employ the cylindrical coordinate system $(R, Z, \phi)$, which is right handed, but unconventional in order [17], and referring to the last subsection,

$$
\begin{aligned}
& \boldsymbol{U}=R \Omega \boldsymbol{e}_{\phi}, \\
& \boldsymbol{U} \cdot \nabla \boldsymbol{\xi}=\Omega\left(i n \boldsymbol{\xi}-\boldsymbol{e}_{Z} \times \boldsymbol{\xi}\right), \\
& \boldsymbol{U} \cdot \boldsymbol{\nabla}(\boldsymbol{U} \cdot \boldsymbol{\nabla} \boldsymbol{\xi})=-n^{2} \Omega^{2} \boldsymbol{\xi}-2 i n \Omega^{2} \boldsymbol{e}_{Z} \times \boldsymbol{\xi}, \\
& (\boldsymbol{U} \cdot \boldsymbol{\nabla}) \boldsymbol{U}=-\Omega^{2} \boldsymbol{R}_{R},
\end{aligned}
$$


where $\boldsymbol{\xi}=\hat{\boldsymbol{\xi}}(\theta, \psi) \exp (i n \phi-i \omega t)$ has been assumed, $\boldsymbol{e}_{Z}=$ $\boldsymbol{\nabla} Z, \boldsymbol{e}_{R}=\boldsymbol{\nabla} R$ and $\boldsymbol{e}_{\phi}=R \boldsymbol{\nabla} \phi$. Clearly, we thus have in the momentum equation $\partial \boldsymbol{\xi} / \partial t=-i \omega \boldsymbol{\xi}$ and $\partial^{2} \boldsymbol{\xi} / \partial t^{2}=$ $-\omega^{2} \boldsymbol{\xi}$. It is then straightforward to show that

$$
\begin{aligned}
- & \rho\left[(\omega-n \Omega)^{2} \boldsymbol{\xi}-2 i(\omega-n \Omega) \Omega \boldsymbol{e}_{Z} \times \boldsymbol{\xi}\right]+\boldsymbol{\nabla} \cdot\left(\rho \Omega^{2} \boldsymbol{\xi}\right) R \boldsymbol{e}_{R} \\
& =(\boldsymbol{\delta} \boldsymbol{B} \cdot \boldsymbol{\nabla}) \boldsymbol{B}+(\boldsymbol{B} \cdot \boldsymbol{\nabla}) \boldsymbol{\delta} \boldsymbol{B}-\boldsymbol{\nabla}(\boldsymbol{\delta} \boldsymbol{B} \cdot \boldsymbol{B})+ \\
& \boldsymbol{\nabla}(\boldsymbol{\xi} \cdot \boldsymbol{\nabla} P+\Gamma P \boldsymbol{\nabla} \cdot \boldsymbol{\xi}) .
\end{aligned}
$$

Note that the last term on the left hand side of the above equation is often put on the right hand side, and considered an applied force. The frequency $\omega-n \Omega \equiv \omega_{D}$ is clearly the mode frequency in the rest frame of the equilibrium plasma. Finally, as will be seen, it is desirable to write the momentum equation in terms of the perturbed pressure $\delta P$. From $\delta P=-\boldsymbol{\xi} \cdot \nabla P-\Gamma P \boldsymbol{\nabla} \cdot \boldsymbol{\xi}$ it is easy to show that

$$
\begin{aligned}
-\rho \omega_{D}^{2} \boldsymbol{\xi} & +\left[\boldsymbol{\xi} \cdot \nabla\left(\rho \Omega^{2}\right)-\rho \frac{\Omega^{2}}{\Gamma P}(\delta P+\boldsymbol{\xi} \cdot \nabla P)\right] R \boldsymbol{e}_{R} \\
& +2 i \rho \omega_{D} \Omega \boldsymbol{e}_{Z} \times \boldsymbol{\xi}+\nabla \delta P \\
& =(\boldsymbol{\delta} \boldsymbol{B} \cdot \nabla) \boldsymbol{B}+(\boldsymbol{B} \cdot \boldsymbol{\nabla}) \boldsymbol{\delta} \boldsymbol{B}-\boldsymbol{\nabla}(\boldsymbol{\delta} \boldsymbol{B} \cdot \boldsymbol{B}) .
\end{aligned}
$$

Following Bussac et al [18] it is often convenient to write the total displacement as

$$
\boldsymbol{\xi}=\boldsymbol{\xi}_{p}+\alpha \boldsymbol{B}
$$

with the property $\boldsymbol{\xi}_{p} \cdot \boldsymbol{\nabla} \phi=0$. Clearly $\alpha B$ is closely related (but not identical) to $\xi_{\|}=\boldsymbol{b} \cdot \boldsymbol{\xi}$, and notations will be interchanged where convenient. In Section III where static (non-rotating) plasmas are considered, it will be shown that zonal modes are exactly electrostatic, and there are no flows across magnetic flux surfaces. While full electromagnetic calculations have been made for GAMs and zonal modes [5], analysis of the continuum mode frequencies in rotating plasma are recovered on setting $\xi_{p}^{\psi}=\boldsymbol{\xi}_{p} \cdot \boldsymbol{\nabla} \psi=0$ and $\boldsymbol{\delta} \boldsymbol{B}=0$ in Eq. (13). Let us now examine the perturbed pressure $\delta P=-\boldsymbol{\xi} \cdot \boldsymbol{\nabla} P-\Gamma P \boldsymbol{\nabla} \cdot \boldsymbol{\xi}$, a quantity that will ultimately be eliminated. With $\xi_{p}^{\psi}=0$, Eq. (8) and Eq. (7), we obtain

$$
\begin{aligned}
\delta P= & -\Gamma P\left[\frac{1}{\mathcal{J}}\left(\frac{\partial}{\partial \theta}+q \frac{\partial}{\partial \phi}\right) \alpha+\nabla \cdot \boldsymbol{\xi}_{p}\right] \\
& -\rho R \Omega^{2}\left(\xi_{p}^{\theta}+\frac{F}{q R^{2}} \alpha\right) \frac{\partial R}{\partial \theta} .
\end{aligned}
$$

where $\xi_{p}^{\theta}=\boldsymbol{\xi}_{p} \cdot \boldsymbol{\nabla} \theta$. We now wish to simplify the perturbed pressure. It can be shown that $\delta B^{\phi}=-\nabla$. $\left(\boldsymbol{\xi}_{p} F / R^{2}\right)$, so that on letting (see earlier discussions) $\delta B^{\phi}=0$ and $\xi_{p}^{\psi}=0$, we have $\boldsymbol{\nabla} \cdot \boldsymbol{\xi}_{p}=\xi_{p}^{\theta} R^{-2} \partial R^{2} / \partial \theta$ to relevant order. At this point it is assumed that all fluctuating quantities adopt the form $X=\hat{X}(\psi, \theta) \exp (i n \phi-$ $i m \theta)$. Choosing the singular location $q=m / n$, the per- turbed pressure is

$$
\begin{aligned}
\hat{\delta P}(\psi, \theta)= & -\rho R\left[\omega_{s}^{2}\left(\frac{F}{q R} \frac{\partial \hat{\alpha}}{\partial \theta}+2 \hat{\xi}_{p}^{\theta} \frac{\partial R}{\partial \theta}\right)\right. \\
& \left.+\Omega^{2}\left(\hat{\xi}_{p}^{\theta}+\frac{F}{q R^{2}} \hat{\alpha}\right) \frac{\partial R}{\partial \theta}\right]\left[1+O\left(\epsilon^{2}\right)\right],
\end{aligned}
$$

with the sound frequency $\omega_{s}^{2}=\Gamma P /\left(\rho R^{2}\right)$, and where $\epsilon$ is the local inverse aspect ratio, such that $R B / F=$ $1+O\left(\epsilon^{2}\right)$. Another important related quantity is the Mach number $M$, which we define as,

$$
M^{2}=\frac{\rho \Omega^{2} R^{2}}{2 P}=\frac{\Gamma \Omega^{2}}{2 \omega_{s}^{2}} .
$$

Now, assuming $M \sim 1$ or less, and $\alpha^{-1} \partial \alpha / \partial \theta \sim O\left(\epsilon^{0}\right)$, the last term in Eq. (14) can be neglected to leading order in $\epsilon$, so that

$$
\hat{\delta P}(\psi, \theta)=-\rho R\left[\omega_{s}^{2}\left(\frac{F}{q R} \frac{\partial \hat{\alpha}}{\partial \theta}+2 \hat{\xi}_{p}^{\theta} \frac{\partial R}{\partial \theta}\right)+\Omega^{2} \hat{\xi}_{p}^{\theta} \frac{\partial R}{\partial \theta}\right] .
$$

Returning to the equation of motion, and dotting Eq. (13) with $\boldsymbol{b}$ (neglecting $\boldsymbol{\xi}_{p} \cdot \boldsymbol{b} \approx R \xi_{p}^{\theta}(\epsilon / q)^{2}$ ), and taking $\boldsymbol{\delta} \boldsymbol{B}=0$, yields:

$$
\begin{aligned}
-\rho \omega_{D}^{2} \alpha B= & -\left[\boldsymbol{\xi} \cdot \nabla\left(\rho \Omega^{2}\right)-\rho \frac{\Omega^{2}}{\Gamma P}(\delta P+\boldsymbol{\xi} \cdot \nabla P)\right] R \boldsymbol{e}_{R} \cdot \boldsymbol{b} \\
& -\boldsymbol{b} \cdot \nabla \delta P-2 i \rho \omega_{D} \Omega\left(\boldsymbol{e}_{Z} \times \boldsymbol{\xi}\right) \cdot \boldsymbol{b} .
\end{aligned}
$$

An initial simplification can be made by noting that the terms in the above equation that are proportional to $\boldsymbol{e}_{R} \cdot \boldsymbol{b}$ are proportional to $(\partial R / \partial \theta)^{2} \sim \epsilon^{2} R^{2}$. Neglecting these higher order (in $\epsilon$ ) terms, using $\boldsymbol{b}$. $\boldsymbol{e}_{Z} \times \boldsymbol{\xi}=-\xi_{p}^{\theta}(F /(R B)) \partial R / \partial \theta, \boldsymbol{b} \cdot \boldsymbol{\nabla} \delta P=\exp ($ in $\phi-$ $i m \theta)(1 /(\mathcal{J} B)) \partial \hat{\delta P} / \partial \theta, R B / F=1+O\left(\epsilon^{2}\right)$ and on substituting Eq. (16), gives to leading order in $\epsilon$ :

$$
\begin{aligned}
q \omega_{D}^{2} \hat{\alpha} B= & \frac{\partial}{\partial \theta}\left[\omega_{s}^{2}\left(\frac{F}{q R} \frac{\partial \hat{\alpha}}{\partial \theta}+2 \hat{\xi}_{p}^{\theta} \frac{\partial R}{\partial \theta}\right)+\Omega^{2} \hat{\xi}_{p}^{\theta} \frac{\partial R}{\partial \theta}\right] \\
& -2 i q \omega_{D} \Omega \frac{\partial R}{\partial \theta} \hat{\xi}_{p}^{\theta} .
\end{aligned}
$$

Now, we expect $\alpha$ to have dominant neighbouring sideband harmonics, so we search for a solution to Eq. (17) in the form

$\hat{\alpha}(\psi, \theta)=\hat{\alpha}^{(-1)}(\psi) \exp (-i \theta)+\hat{\alpha}^{(0)}(\psi)+\hat{\alpha}^{(+1)}(\psi) \exp (i \theta)$,

With $R=R_{0}(1+\epsilon \cos \theta)$ and $R_{0}$ the radius of the magnetic axis, such that $F=R_{0} B_{0}\left(1+O\left(\epsilon^{2}\right)\right)$ where $B_{0}$ is the magnetic field on the magnetic axis, then $\partial R / \partial \theta=-\left(\epsilon R_{0} / 2\right)[\exp (i \theta)+\exp (-i \theta)]$. Substituting $\hat{\alpha}$ into Eq. (17) and Fourier analysing gives,

$$
\begin{aligned}
\hat{\alpha}^{(0)} & =0 \\
\omega_{D}^{2} q B_{0} \hat{\alpha}^{(1)} & =\frac{\omega_{s}^{2}}{q} \hat{\alpha}^{(1)} B_{0}+\epsilon R_{0} \hat{\xi}_{p}^{\theta}\left(\omega_{s}^{2}+\frac{\Omega^{2}}{2}+q \omega_{D} \Omega\right) \\
\omega_{D}^{2} q B_{0} \hat{\alpha}^{(-1)} & =\frac{\omega_{s}^{2}}{q} \hat{\alpha}^{(-1)} B_{0}+\epsilon R_{0} \hat{\xi}_{p}^{\theta}\left(\omega_{s}^{2}+\frac{\Omega^{2}}{2}-q \omega_{D} \Omega\right),
\end{aligned}
$$


so that to leading order in $\epsilon$ the parallel displacement has no flute component. Substituting these results into $\hat{\alpha}$ succinctly gives to leading order in $\epsilon$ :

$$
\hat{\alpha} B_{0}=-\frac{2 q \epsilon R_{0} \hat{\xi}_{p}^{\theta}}{\omega_{s}^{2}-q^{2} \omega_{D}^{2}}\left[\left(\omega_{s}^{2}+\frac{\Omega^{2}}{2}\right) \cos \theta+q \omega_{D} \Omega i \sin \theta\right],
$$

where the $\Omega^{2} \cos \theta$ term arrives due to centrifugal effects, and the $\omega_{D} \Omega \sin \theta$ term arrives due to the coriolis effect. Both centrifugal and coriolis effects ultimately influence the continua, as will be seen. Finally, substitution of Eq. (18) into Eq. (16) yields the lowest order pressure fluctuation:

$$
\begin{aligned}
\hat{\delta P}=-\frac{\rho R_{0}^{2} \epsilon \hat{\xi}_{p}^{\theta}}{\omega_{s}^{2}-q^{2} \omega_{D}^{2}} & {\left[q^{2} \omega_{D}^{2}\left(2 \omega_{s}^{2}+\Omega^{2}\right) \sin \theta\right.} \\
& \left.-2 q \omega_{D} \Omega \omega_{s}^{2} i \cos \theta\right] .
\end{aligned}
$$

We are now ready to obtain the continua, which are conveniently obtained by applying a vorticity operator (see Refs. [19, 20]):

$$
V(\boldsymbol{X})=\frac{1}{2 \pi} \int_{-\pi}^{\pi} \frac{d \theta i \exp (i m \theta)}{m} q|\nabla \psi| \mathcal{J} \nabla \cdot\left[\frac{\boldsymbol{X} \times \boldsymbol{\nabla} \phi}{\boldsymbol{B} \cdot \boldsymbol{\nabla} \phi}\right] .
$$

on the equation of motion $\boldsymbol{X}(\psi, \theta, \phi)$, where $\boldsymbol{X}$ is Eq. (13). On letting $\boldsymbol{X}$ take the form $\boldsymbol{X}=\boldsymbol{Y}+\boldsymbol{\nabla} \delta P$ with $\boldsymbol{Y}=Y_{\psi} \boldsymbol{\nabla} \psi+Y_{\theta} \boldsymbol{\nabla} \theta+Y_{\phi} \boldsymbol{\nabla} \phi$ one then solves

$$
\begin{array}{r}
V=\frac{q|\boldsymbol{\nabla} \psi|}{i m} \frac{1}{2 \pi} \int_{\pi}^{\pi} d \theta\left\{\frac{\partial}{\partial \psi}\left[\hat{\delta P} \frac{\partial}{\partial \theta}\left(\frac{\mathcal{J}}{q}\right)-\left(\frac{\mathcal{J}}{q}\right) \hat{Y}_{\theta}\right]\right. \\
\left.+i m\left[\delta \hat{P} \frac{\partial}{\partial \psi}\left(\frac{\mathcal{J}}{q}\right)-\left(\frac{\mathcal{J}}{q}\right) \hat{Y}_{\psi}\right]\right\}=0
\end{array}
$$

where to leading order (neglecting non-orthogonality of coordinates at the required order in $\epsilon$ ):

$$
\begin{aligned}
\hat{Y}_{\psi}= & |\boldsymbol{\nabla} \psi|^{-2}\left[-\rho \omega_{D}^{2} \hat{\boldsymbol{\xi}}+\left(\hat{\boldsymbol{\xi}} \cdot \boldsymbol{\nabla}\left(\rho \Omega^{2}\right)-\right.\right. \\
& \left.\left.\rho \frac{\Omega^{2}}{\Gamma P}(\hat{P}+\hat{\boldsymbol{\xi}} \cdot \boldsymbol{\nabla} P)\right) R \boldsymbol{e}_{R}+2 i \rho \omega_{D} \Omega \boldsymbol{e}_{Z} \times \hat{\boldsymbol{\xi}}\right] \cdot \nabla \psi, \\
\hat{Y}_{\theta}= & |\boldsymbol{\nabla} \theta|^{-2}\left[-\rho \omega_{D}^{2} \hat{\boldsymbol{\xi}}+\left(\hat{\boldsymbol{\xi}} \cdot \boldsymbol{\nabla}\left(\rho \Omega^{2}\right)-\right.\right. \\
& \left.\left.\rho \frac{\Omega^{2}}{\Gamma P}(\hat{P}+\hat{\boldsymbol{\xi}} \cdot \boldsymbol{\nabla} P)\right) R \boldsymbol{e}_{R}+2 i \rho \omega_{D} \Omega \boldsymbol{e}_{Z} \times \hat{\boldsymbol{\xi}}\right] \cdot \nabla \theta .
\end{aligned}
$$

It turns out that the expression in the second set of square parenthesis in Eq. ( 21 ) is small, especially for $\xi_{p}^{\psi}=0$, and will be neglected henceforth. Thus, it is sufficient to solve,

$$
\int_{\pi}^{\pi} d \theta\left[\hat{\delta P} \frac{\partial}{\partial \theta}\left(\frac{\mathcal{J}}{q}\right)-\left(\frac{\mathcal{J}}{q}\right) \hat{Y}_{\theta}\right]=0
$$

which in the notation employed in Ref. [5], coincides with
$A_{1}=0$. Letting $\hat{Y}_{\theta}=\hat{Y}_{1}+\hat{Y}_{2}+\hat{Y}_{3}+\hat{Y}_{4}$ we may write:

$$
\begin{aligned}
& \hat{Y}_{1}=|\boldsymbol{\nabla} \theta|^{-2}\left(-\rho \omega_{D}^{2} \hat{\boldsymbol{\xi}}\right) \cdot \boldsymbol{\nabla} \theta \\
& \hat{Y}_{2}=|\boldsymbol{\nabla} \theta|^{-2}\left[\hat{\boldsymbol{\xi}} \cdot \boldsymbol{\nabla}\left(\rho \Omega^{2}\right)\right] R \boldsymbol{\nabla} R \cdot \boldsymbol{\nabla} \theta \\
& \hat{Y}_{3}=|\boldsymbol{\nabla} \theta|^{-2}\left[-\rho \frac{\Omega^{2}}{\Gamma P}(\delta \hat{P}+\hat{\boldsymbol{\xi}} \cdot \boldsymbol{\nabla} P)\right] R \boldsymbol{\nabla} R \cdot \boldsymbol{\nabla} \theta \\
& \hat{Y}_{4}=|\boldsymbol{\nabla} \theta|^{-2}\left(2 i \rho \omega_{D} \Omega \boldsymbol{e}_{Z} \times \hat{\boldsymbol{\xi}}\right) \cdot \boldsymbol{\nabla} \theta .
\end{aligned}
$$

Using results already stated above (e.g. that $\nabla R \cdot \nabla \theta=$ $\left.|\nabla \theta|^{2} \partial R / \partial \theta\right)$, and with $\omega_{A}^{2}=B^{2} /\left(\rho R^{2}\right)$, then at leading order,

$$
\begin{aligned}
-\left(\frac{\mathcal{J}}{q}\right) \hat{Y}_{1}= & \frac{r^{2} B_{0}}{R_{0}} \xi_{p}^{\theta} \frac{\omega_{D}^{2}}{\omega_{A}^{2}} \\
-\left(\frac{\mathcal{J}}{q}\right) \hat{Y}_{2}= & \frac{r^{2} B_{0}}{R_{0}} \xi_{p}^{\theta} \frac{\Omega^{2} M^{2}}{\omega_{A}^{2}}\left(-2 \sin ^{2} \theta\right) \\
-\left(\frac{\mathcal{J}}{q}\right) \hat{Y}_{3}= & \frac{r^{2} B_{0}}{R_{0}} \xi_{p}^{\theta} \frac{\Omega^{2}\left(\Omega^{2}+2 q^{2} \omega_{D}^{2}\right)}{\omega_{A}^{2}\left(\omega_{s}^{2}-q^{2} \omega_{D}^{2}\right)} \sin ^{2} \theta \\
-\left(\frac{\mathcal{J}}{q}\right) \hat{Y}_{4}= & \frac{r^{2} B_{0}}{R_{0}} \xi_{p}^{\theta} \frac{q^{2} \omega_{D}^{2} \Omega^{2}}{\omega_{A}^{2}\left(\omega_{s}^{2}-q^{2} \omega_{D}^{2}\right)} \times \\
& 4\left[\sin ^{2} \theta+\frac{i}{q \omega_{D} \Omega}\left(\omega_{s}^{2}+\frac{\Omega^{2}}{2}\right) \sin \theta \cos \theta\right] .
\end{aligned}
$$

It is seen that the coriolis effect will survive through the $\sin ^{2} \theta$ contribution in $\hat{Y}_{4}$, while all other $\Omega^{2}$ contributions in $\hat{Y}$ are due to centrifugal effects. Centrifugal effects also arise in the remaining term of the vorticity, namely,

$$
\begin{aligned}
\hat{\delta P} & \frac{\partial}{\partial \theta}\left(\frac{\mathcal{J}}{q}\right)=\frac{r^{2} B_{0}}{R_{0}} \xi_{p}^{\theta} \times \\
& \frac{\left[q^{2} \omega_{D}^{2}\left(2 \omega_{s}^{2}+\Omega^{2}\right) \sin ^{2} \theta-2 i q \omega_{D} \Omega \omega_{s}^{2} \cos \theta \sin \theta\right]}{\omega_{A}^{2}\left(\omega_{s}^{2}-q^{2} \omega_{D}^{2}\right)},
\end{aligned}
$$

where Eq. (19) and $\partial \mathcal{J} / \partial \theta \approx-\left(2 r / B_{0}\right) \sin \theta$ has been used. The coriolis contribution in the above equation (proportionial to $-2 i q \omega_{D} \Omega \omega_{s}^{2} \sin \theta \cos \theta$ ) will not survive integration over $\theta$ in the vorticity of Eq. (22). In the limit of vanishing $\Omega$, standard ideal MHD results are obtained, such that continua comprise only contributions from $\hat{Y}_{1}$ and the first term in the equation for $\hat{\delta P \partial}(\mathcal{J} / q) / \partial \theta$ above.

Finally substituting these results into Eq. (22) gives the continuum equation:

$$
\frac{\omega_{D}^{2}-\Omega^{2} M^{2}}{\omega_{A}^{2}}+\frac{\Omega^{4}+4 q^{2} \omega_{D}^{2} \omega_{s}^{2}\left(1+4 M^{2} / \Gamma\right)}{2 \omega_{A}^{2}\left(\omega_{s}^{2}-q^{2} \omega_{D}^{2}\right)}=0,
$$

where the definition (15) for the Mach number $M$ has again been used. Now, clearly, Eq. (23) has two roots for $\omega_{D}^{2}$. The low frequency root, or zonal root, under the limit $\omega_{D}<<\Omega$, is

$$
\omega_{D}^{2}(\text { zonal })=\frac{1}{1+2 q^{2}+8 q^{2} M^{2} / \Gamma} \Omega^{2} M^{2}\left(1-\frac{1}{\Gamma}\right) .
$$


The high frequency root, or GAM root, is easily obtained in the limit of $M<<1$, or specifically for $\omega_{D}^{2} \sim \omega_{s}^{2} \gg$ $\Omega^{2} M^{2}$, for which one obtains the small rotation correction to the GAM frequency,

$$
\omega_{D}^{2}(\mathrm{GAM})=\omega_{s}^{2}\left[\frac{1}{q^{2}}+2\left(1+\frac{4 M^{2}}{\Gamma}\right)\right] .
$$

This subsection has reviewed continuum modes in rotating plasmas with finite (or zero) mode number $n$, and has hopefully provided a clear passage for obtaining high and low frequency continua. Since it is now clear that the low frequency mode of Eq. (23) is a true continuum mode, whose frequency diminishes to zero as $\Omega$ vanishes, a new calculation can confidently be commenced in static plasmas, where a zonal mode with zero mode frequency is sought, and an exact calculation for the mode vector provided.

\section{EXACT ZONAL MODES IN STATIC IDEAL MHD PLASMAS}

We are now ready to solve the zonal mode problem in static plasmas. While in the last section magnetic fluctuations were assumed small, and perturbed flows (or displacements) were assumed to exist only on rational surfaces, it will be shown below that for a static equilibrium plasma $(\Omega=0)$, the $\omega=0$ zonal mode is exactly electrostatic, and cross field flows are exactly zero. Since one normally thinks of zonal flows, rather than zonal displacements, and since we expect to obtain $\omega=0$, it is more intuitive to write the MHD equations in terms of perturbed velocity $\boldsymbol{\delta} \boldsymbol{U}$ than in terms of displacements. Linearising the MHD equations in the absence of equilibrium flow, one obtains

$$
\begin{aligned}
-\rho \omega^{2} \boldsymbol{\delta} \boldsymbol{U} & =\boldsymbol{j} \times \dot{\boldsymbol{\delta}} \dot{\boldsymbol{B}}+\dot{\boldsymbol{\delta}} \times \boldsymbol{B}-\boldsymbol{\nabla} \dot{\bar{P}} \\
\boldsymbol{\delta} \dot{\boldsymbol{B}} & =\boldsymbol{\nabla} \times(\boldsymbol{\delta} \boldsymbol{U} \times \boldsymbol{B}) \\
\dot{\boldsymbol{\delta} \boldsymbol{j}} & =\nabla \times \boldsymbol{\delta} \dot{B} \\
\dot{\delta P} & =-\boldsymbol{\delta} \boldsymbol{U} \cdot \nabla P-\Gamma P \nabla \cdot \boldsymbol{\delta} \boldsymbol{U} \\
\nabla \cdot \dot{\boldsymbol{\delta}} \boldsymbol{B} & =0
\end{aligned}
$$

where $\dot{x}=\partial x / \partial t$ and $\partial^{2} \boldsymbol{\delta} / \partial t^{2}=-\omega^{2} \boldsymbol{\delta} \boldsymbol{U}$ (following from normal modes $\sim \exp (-i \omega t)$, and where Ohm's law $\boldsymbol{\delta} \boldsymbol{E}+\boldsymbol{\delta} \boldsymbol{U} \times \boldsymbol{B}=0$ has been applied.

In the work of Ref. [5], electromagnetic effects are selfconsistently considered in order to obtain global GAMs and zonal flows. However, as already seen, calculation of the continuum mode frequencies do not require magnetic fluctuations. In addition, it will be seen here that in the absence of toroidal rotation, the zonal branch is exactly electrostatic. It should thus be possible to see how the magnetic fluctuations can set to zero via identifying the correct velocity fluctuation. To this end, it is instructive to write down the contravariant magnetic field components in terms of the contravariant velocity fluctuations $\delta U^{\psi}=\boldsymbol{\delta} \boldsymbol{U} \cdot \nabla \psi, \delta U^{\theta}=\boldsymbol{\delta} \boldsymbol{U} \cdot \nabla \theta$ and $\delta U^{\phi}=\boldsymbol{\delta} \boldsymbol{U} \cdot \boldsymbol{\nabla} \phi$ for a straight field line coordinate system with jacobian $\mathcal{J}=q R^{2} / F$ (as will be assumed again henceforth):

$$
\begin{aligned}
\delta \dot{B}^{\psi} & =\frac{1}{\mathcal{J}}\left[i n q+\frac{\partial}{\partial \theta}\right] \delta U^{\psi}, \\
\dot{\delta B} & =-\frac{1}{\mathcal{J}}\left[\frac{\partial \delta U^{\psi}}{\partial \psi}+i n\left(\delta U^{\phi}-q \delta U^{\theta}\right)\right], \\
\dot{\delta} \dot{B}^{\phi} & =-\frac{1}{\mathcal{J}}\left[\frac{\partial}{\partial \psi}\left(q \delta U^{\psi}\right)+\frac{\partial}{\partial \theta}\left(q \delta U^{\theta}-\delta U^{\phi}\right)\right],
\end{aligned}
$$

where modes have toroidal dependence $\sim \exp ($ in $\phi)$, which is of course exact for this linear system, perturbed about an axisymmetric equilibrium. Note that Bussac's [18] choice of displacement $\boldsymbol{\xi}=\boldsymbol{\xi}_{p}+\alpha \boldsymbol{B}$ (or indeed velocity) is no longer convenient, nor necessary. It is seen that $\dot{\delta B}$ and $\dot{\delta B} \dot{B}^{\theta}$ can be set simultaneously to zero only if $\delta U^{\psi}=0$, i.e. that there are no flows across flux surfaces. It remains to obtain the flow components $\delta U^{\phi}$ and $\delta U^{\theta}$, together with $\delta U_{\phi}$ and $\delta U_{\theta}$, these latter two components defining the parallel velocity,

$$
\delta U_{\|}=\boldsymbol{\delta} \boldsymbol{U} \cdot \boldsymbol{b}=\frac{1}{B \mathcal{J}}\left(q \delta U_{\phi}+\delta U_{\theta}\right)
$$

where the covariant velocity components are defined via $\boldsymbol{\delta} \boldsymbol{U}=\delta U_{\phi} \boldsymbol{\nabla} \phi+\delta U_{\theta} \boldsymbol{\nabla} \theta+\delta U_{\psi} \boldsymbol{\nabla} \psi$. For $\delta U^{\psi}=0$, the parallel velocity can be written simply in terms of the contravariant velocities:

$$
\delta U_{\|}=\frac{F}{B}\left[\delta U^{\phi}+q\left(\frac{R^{2} B^{2}}{F^{2}}-1\right) \delta U^{\theta}\right],
$$

where $\delta U_{\phi}=R^{2} \delta U^{\phi}, \quad \delta U_{\theta}=(q R / F)^{2} \nabla \psi^{2} \delta U^{\theta}$ and $\delta U_{\psi}=-(q R / F)^{2}(\nabla \theta \cdot \nabla \psi) \delta U^{\theta}$.

Finally, with $\delta U^{\psi}=0$, then Eq. (28) yields $\delta \dot{P}=$ $-\Gamma P \nabla \cdot \boldsymbol{\delta} \boldsymbol{U}$. Thus, both the equation of motion and the adiabatic equation of state are then satisfied by $\omega^{2}=0$ and $\boldsymbol{\nabla} \cdot \boldsymbol{\delta} \boldsymbol{U}=0$. To summarise, the ideal MHD model of Eqs. (25) - (29) hold exactly for

$$
\begin{aligned}
& \omega=0, \quad \delta U^{\psi}=0, \quad n\left(\delta U^{\phi}-q \delta U^{\theta}\right)=0, \\
& \frac{\partial \delta U^{\phi}}{\partial \theta}=q \frac{\partial \delta U^{\theta}}{\partial \theta}, \quad \frac{1}{\mathcal{J}}\left[\frac{\partial}{\partial \theta}\left(\mathcal{J} \delta U^{\theta}\right)+i n \mathcal{J} \delta U^{\phi}\right]=0,
\end{aligned}
$$

where we are reminded that a mode with $\omega=0$ is the zonal continuum mode obtained in Eq. (24) for vanishing toroidal equilibrium rotation. The last identity in the above follows from $\boldsymbol{\nabla} \cdot \boldsymbol{\delta} \boldsymbol{U}=0$, while the second, third and fourth identities follow from the respective electrostatic identities of Eq. (30). Clearly, to further explore the spatial dependence of these zonal modes, one must first specify the toroidal mode number of interest.

\section{A. Axisymmetric Zonal Modes}

On choosing axisymmetric $(n=0)$ zonal modes, Eq. (32) requires that $\partial\left(\mathcal{J} \delta U^{\theta}\right) / \partial \theta=0$. With $\mathcal{J}=q R^{2} / F$, 
then it follows that

$$
\delta U^{\theta}(\psi, \theta)=\delta \tilde{U}^{\theta}(\psi) \frac{R_{0}^{2}}{R^{2}}
$$

with $\delta \tilde{U}^{\phi}(\psi)$ some arbitrary function of $\psi$. In addition, from Eq. (32), $\partial \delta U^{\phi} / \partial \theta=q \partial \delta U^{\theta} / \partial \theta$ can be integrated in $\theta$ to yield,

$\delta U^{\phi}(\psi, \theta)=q \delta U^{\theta}(\psi, \theta)+\delta \tilde{U}^{\phi}(\psi)=q \delta \tilde{U}^{\theta}(\psi) \frac{R_{0}^{2}}{R^{2}}+\delta \tilde{U}^{\phi}(\psi)$

where the presently arbitrary constant of integration $\delta \tilde{U}^{\phi}(\psi)$ is related to Ferraro's [21] law of isorotation. Now, true $n=0$ zonal flows do not propagate secularly in the toroidal direction [1]. The flow forms a closed circuit, or convective cell, over one poloidal circuit, which requires that $\delta \tilde{U}^{\phi}(\psi)=-q \delta \tilde{U}^{\theta}(\psi)$. Hence, in summary,

$\delta U^{\theta}(\psi, \theta)=\delta \tilde{U}^{\theta}(\psi) \frac{R_{0}^{2}}{R^{2}}$, and $\delta U^{\phi}=q \delta \tilde{U}^{\theta}(\psi)\left(\frac{R_{0}^{2}}{R^{2}}-1\right)$.

Finally, consider the perturbed electric field, which from Ohm's law (assuming $\delta U^{\psi}=0$ ), is

$$
\boldsymbol{\delta} \boldsymbol{E}=-q\left(\delta U^{\theta}-\frac{\delta U^{\phi}}{q}\right) \nabla \psi
$$

Thus the $n=0$ zonal mode has, on choosing that the flow closes on itself, an associated electric field $\boldsymbol{\delta} \boldsymbol{E}=$ $-q \delta \tilde{U}^{\theta}(\psi) \nabla \psi$ with no poloidal dependence.

\section{B. Non-axisymmetric Zonal Modes}

We now turn to non-axisymmetric zonal modes. Equation (32) for $n \neq 0$ requires that

$$
\delta U^{\phi}=q \delta U^{\theta}(\psi, \theta)
$$

i.e. the same result as for $n=0$ zonal modes, except that for $n \neq 0$, there is no isorotation contribution $\delta \tilde{U}^{\phi}(\psi)$. It is thus seen from Eq. (33) that the electric field $\delta E$ is identically zero for $n \neq 0$ zonal flows, so that in addition $\boldsymbol{\delta} \boldsymbol{U}=\delta U_{\|} \boldsymbol{b}$. Since electric fields and magnetic perturbations are zero for the $n \neq 0$ case, then non-axisymmetric zonal modes are strictly hydrodynamic phenomena.

Let us now investigate the exact spatial dependence of the $n \neq 0$ zonal velocity. Substituting $\delta U^{\phi}=q \delta U^{\theta}(\psi, \theta)$ into the $\boldsymbol{\nabla} \cdot \boldsymbol{\delta} \boldsymbol{U}=0$ (the last relation in Eq. (32), we find that (using $\mathcal{J}=q R^{2} / F$ ):

$$
\frac{\partial}{\partial \theta}\left(R^{2} \delta U^{\theta}\right)+i n q R^{2} \delta U^{\theta}=0 .
$$

The solution is necessarily $\delta U^{\theta}=\left[f(\psi) / R^{2}\right] \exp ($ in $\phi-$ inq $\theta$ ), with $f(\psi)$ an arbitrary function of $\psi$. Noting however, that $\boldsymbol{\delta} \boldsymbol{U}$ must be periodic in $\theta$, the solution requires that $n q$ is an integer, i.e. $n q(\psi)=m$ with $m$ integer. Any particular $(n, m)$ mode can exist only at its own rational surface $q(\psi)=m / n$, so that summarising,

$$
\begin{aligned}
\delta U^{\theta}(\psi, \theta, \phi) & =\delta \tilde{U} \delta\left(\psi-\psi_{m, n}\right)\left[\frac{R_{0}}{R(\psi, \theta)}\right]^{2} \exp (i n \phi-i m \theta), \\
\delta U^{\phi} & =\frac{m}{n} \delta U^{\theta},
\end{aligned}
$$

where $\delta \tilde{U}$ is an arbitrary coefficient, $\delta\left(\psi-\psi_{m, n}\right)$ is a Dirac delta function, and $q\left(\psi=\psi_{m, n}\right)=m / n$. The plasma has of course an infinite number of rational surfaces, so there ia an infinite spectrum of these singularly localised (in $\psi$ ) non-axisymmetric zonal modes. A final point to note on the general structure of the $n \neq 0$ zonal flows is that since the flow follows exactly the direction of the magnetic field, and since the flow is confined to an exact magnetic surface where $q=m / n$, then the flow exactly closes on itself. Thus, while $n=0$ and $n \neq 0$ zonal modes differ in that $n=0$ modes have finite perpendicular velocity, and associated radial electric fields, both $n=0$ and $n \neq 0$ flows close on themselves and thus can be considered convective cells.

Let us now examine the spatial dependence of the $n \neq 0$ zonal mode using inverse aspect ratio analytic expansion of the equilibrium. In particular, since the exact poloidal dependence of the flow vectors is known, we can calculate the amplitudes of sideband harmonics relative to a main harmonic $\delta U(\psi) \exp (-i m \theta)$. For example, taking a leading order inverse aspect ratio $(\epsilon)$ expansion for $R$, one obtains,

$$
\begin{aligned}
& \delta U^{\theta}(\psi, \theta, \phi) \approx \delta \tilde{U} \delta\left(\psi-\psi_{m, n}\right) \exp (i n \phi) \times \\
& \{-\epsilon \exp [-i(m-1) \theta]+\exp [-i m \theta]-\epsilon \exp [-i(m+1) \theta]\}
\end{aligned}
$$

Finally, from Eq. (31), it is also possible to write the total fluid perturbation as

$$
\begin{aligned}
\boldsymbol{\delta} \boldsymbol{U} & =\frac{q R^{2} B}{F} \delta U^{\theta} \boldsymbol{b} \\
& =\frac{q R_{0} B(\theta) \boldsymbol{b}}{F} \delta \tilde{U} \delta\left(\psi-\psi_{m, n}\right) \exp (i n \phi-i m \theta),
\end{aligned}
$$

where it is again straightforward to obtain the sideband harmonics. Higher order corrections are easily calculated, so that elongation, triangularity and toroidicity (Shafranov shift) effects on the sidebands are tractable.

\section{EXACT ZONAL MODES UNDER KINETIC-MHD MODEL}

The problem in the last section is modified to include kinetic effects. As before, toroidal rotation in the equilibrium is neglected. Equations (25) - (29) are replaced 
with [22]:

$$
\begin{aligned}
& -i \sum_{j} \rho_{j}\left(\omega-n \omega_{* p j}\right) \dot{\boldsymbol{\delta}} \dot{\boldsymbol{U}}_{\perp}=\boldsymbol{j} \times \dot{\boldsymbol{\delta}} \dot{\boldsymbol{B}}+\dot{\boldsymbol{\delta}} \boldsymbol{j} \times \boldsymbol{B}+ \\
& \left(\dot{\delta} \dot{P}_{\perp}-\dot{\delta} \dot{P}_{\|}\right) \kappa-\nabla_{\perp} \dot{\delta} \dot{P}_{\perp} \\
& \sum_{j} q_{j} \delta \dot{n}_{j}=0 \\
& \dot{\boldsymbol{B}}=\nabla \times\left(\boldsymbol{\delta} \boldsymbol{U}_{\perp A} \times \boldsymbol{B}\right) \\
& \dot{\delta} \boldsymbol{j}=\nabla \times \dot{\delta} \dot{B} \\
& \dot{\delta f_{j}}=q_{j} R^{2} \dot{\boldsymbol{\delta}} \dot{\phi} \frac{\partial F_{j}}{\partial \mathcal{P}_{\phi}}+q_{j} \dot{\delta \phi} \frac{\partial F_{j}}{\partial \mathcal{E}}-\frac{\mu \dot{\delta} \dot{B}_{\|}}{B} \frac{\partial F_{j}}{\partial \mu}+\dot{\delta f_{j k}}, \\
& \left(-i \omega+\boldsymbol{V}_{j g} \cdot \nabla\right) \delta \dot{f}_{j k}=-i \delta \dot{L}_{j}\left(\omega \frac{\partial F_{j}}{\partial \mathcal{E}}-n \frac{\partial F_{j}}{\partial \mathcal{P}_{\phi}}\right) \\
& \nabla \cdot \boldsymbol{\delta} \dot{B}=0,
\end{aligned}
$$

where $\dot{X} \equiv \partial X / \partial t, q_{j}$ the species charge, $\omega_{* p j}$ is the fluid diamagnetic frequency [23] for species $j$, while $\delta n_{j}$, $\delta P_{j \perp}$ and $\delta P_{j \|}$ are obtained by solving the drift kinetic equation (38) for $\delta f_{j}$ (the perturbed distribution function for each species $j$ ) in terms of the electromagnetic fields and taking the relevant moments, as described later. In addition, $F_{j}$ is the equilibrium distribution function for the species $j, \mathcal{P}_{\phi}$ and $\mathcal{E}$ are the toroidal canonical momenta and kinetic energy of a particle. Finally, $\boldsymbol{V}_{j g}$ is the guiding centre velocity for a particle of species $j$, which also enters into the time derivative of the perturbed Lagrangian, defined as [24]:

$$
\dot{\delta L}=q_{j} \dot{\boldsymbol{A}} \cdot \boldsymbol{V}_{j g}-q_{i} \dot{\delta} \phi-\mu \dot{\delta} \dot{B}_{\|}
$$

where $\mu$ is the magnetic moment, and $\boldsymbol{\delta} \boldsymbol{A}$ and $\delta \phi$ are associated with the perturbed electric field $\boldsymbol{\delta} \boldsymbol{E}=-\boldsymbol{\nabla} \delta \phi-$ $\boldsymbol{\delta} \boldsymbol{A}$ as discussed in more detail below.

There are a few additional points to note before progress is made. The first is that, as discussed further below, in kinetic-MHD, only the perpendicular components of the momentum equation are considered. The parallel momentum equation of ideal MHD is essentially replaced by the quasi-neutrality equation of Eq. (35), which solves for the potential $\delta \phi$ that describes the parallel electric field $\boldsymbol{\delta} \boldsymbol{E} \cdot \boldsymbol{b}=-\boldsymbol{b} \cdot \boldsymbol{\nabla} \delta \phi$. Note also in the set of equations (34) - (39) that there are two types of velocity perturbations, the relation between them is now described. With the gauge $\boldsymbol{b} \cdot \boldsymbol{\delta} \boldsymbol{A}=0$, it can be seen from the general perturbed electric field

$$
\boldsymbol{\delta} \boldsymbol{E}=-\nabla \delta \phi-\dot{\boldsymbol{A}}
$$

that $\boldsymbol{\delta} \boldsymbol{E} \cdot \boldsymbol{b}=-\boldsymbol{b} \cdot \boldsymbol{\nabla} \delta \phi$. In addition, the gauge allows the vector potential to be written as

$$
\dot{\boldsymbol{\delta} \boldsymbol{A}}=\boldsymbol{\delta} \boldsymbol{U}_{\perp A} \times \boldsymbol{B},
$$

which thus explains that the definition of Faraday's law given in Eq. (36) is written in terms of the special velocity perturbation $\boldsymbol{\delta} \boldsymbol{U}_{\perp A}$, where one is free to allow $\boldsymbol{b} \cdot \boldsymbol{\delta} \boldsymbol{U}_{\perp A}=0$. Meanwhile, the left hand side of the perpendicular momentum equation depends on the total perturbed E-cross-B velocity, which from Eqs. (41) and (42) is

$$
\boldsymbol{\delta} \boldsymbol{U}_{\perp}=-\frac{\boldsymbol{\nabla} \delta \phi \times \boldsymbol{B}}{B^{2}}+\boldsymbol{\delta} \boldsymbol{U}_{\perp A},
$$

where for the ideal MHD case $E_{\|}=0$, we clearly have $\boldsymbol{\delta} \boldsymbol{U}_{\perp}=\boldsymbol{\delta} \boldsymbol{U}_{\perp A}$ due to $\delta \phi$ being zero.

The total perturbed velocity for species $j$ is

$$
\boldsymbol{\delta} \boldsymbol{U}_{j}=\boldsymbol{\delta} \boldsymbol{U}_{\perp}+\delta U_{\|} \boldsymbol{b}
$$

where, the perpendicular perturbed velocity $\boldsymbol{\delta} \boldsymbol{U}_{\perp}$ is common to all particle species owing to it being the E-cross-B flow. The parallel perturbed velocity is species dependent, but the crucial point to reiterate is that the linear kinetic-MHD stability problem is independent of $\delta U_{j \|}$. Instead of solving the parallel momentum equation of ideal MHD, kinetic MHD instead treats the parallel dynamics intrinsic to the drift-kinetic equation, which is manifested in (34) - (39) via the perturbed pressure components, and in the $\delta \phi$ contribution to $\delta \boldsymbol{U}_{\perp}$. Precisely, the parallel component of the momentum equation and the adiabatic equation of state both employed in the ideal model are replaced in the drift kinetic model by the drift kinetic equation and quasi-neutrality. Nevertheless, if knowledge of $\delta U_{j \|}$ is desired, then after the kinetic-MHD equations have been solved, the continuity equation for each species $j$,

$$
\frac{\partial \delta \rho_{j}}{\partial t}+\rho_{j} \boldsymbol{\nabla} \cdot \boldsymbol{\delta} \boldsymbol{U}_{j}+\boldsymbol{\delta} \boldsymbol{U}_{j} \cdot \boldsymbol{\nabla} \rho_{j}=0
$$

valid to all orders in Larmor radius, can be inverted, to yield $\delta U_{j \|}$ in terms of the known variables $\delta U_{\perp}$ and $\delta \rho_{j}$. We will do this at the end of this section for the zonal modes under investigation here.

\section{A. Axisymmeteric zonal modes under kinetic-MHD model}

In this section it is shown that the $n=0$ zonal modes derived using the MHD model are exact. Let us first assume the ideal MHD fields, $E_{\|}=0$ and $\boldsymbol{\delta} \boldsymbol{B}=0$, in Eqs. (34) - (39) to see if the kinetic model then exactly recovers ideal MHD. Clearly, as seen from Eq. (43), with $\delta \phi=0$ the two definitions of perturbed velocity are conveniently identical, i.e. $\boldsymbol{\delta} \boldsymbol{U}_{A}=\boldsymbol{\delta} \boldsymbol{U}$. Hence $\boldsymbol{\delta} \boldsymbol{B}=\boldsymbol{\nabla} \times(\boldsymbol{\delta} \boldsymbol{U} \times \boldsymbol{B})$ as is the case for the ideal MHD model, so that again on assuming ideal MHD fields, $\delta U^{\psi}$ should initially be taken to be zero.

We now consider the drift kinetic equation of Eq. (38), which must be treated carefully. We should not yet set $\omega=0$ in the equation for $\delta f_{j k}$ in Eq. (38) because both left and right hand sides contain $\omega$ and $n$, and both sides might vanish (on setting $\omega=0, n=0$ ) if an improper treatment of the problem is made. It is first noted that 
with $\boldsymbol{\delta} \boldsymbol{A}=\boldsymbol{\delta} \boldsymbol{U} \times \boldsymbol{B}$, the perturbed Lagrangian of Eq. (40) is [25]:

$$
\begin{aligned}
\dot{\delta L}= & -q_{j} \delta U^{\psi} \boldsymbol{V}_{j g} \cdot(\boldsymbol{\nabla} \boldsymbol{\phi}-q \boldsymbol{\nabla} \theta) \\
& -q_{j} \delta U^{\theta} \boldsymbol{V}_{j g} \cdot \boldsymbol{\nabla} \boldsymbol{\psi}-q_{j} \dot{\delta \phi}-\mu \dot{\delta} \dot{B}_{\|},
\end{aligned}
$$

so that with $\delta B_{\|}=0, \delta \phi=0$ and $\delta U^{\psi}=0$, we have $\dot{\delta L}=-q_{j} \delta U^{\theta} \boldsymbol{V}_{j g} \cdot \boldsymbol{\nabla} \boldsymbol{\psi}$. The associated drift kinetic problem was treated in the appendix of Ref. [23], where it was found that on assuming $\omega$ much less than the bounce frequency, the perturbed distribution function adopts the form $\dot{\delta f} f_{j k}(\psi, \theta, \phi, t)=\delta \hat{\dot{f}}_{j k}(\psi, \theta) \exp (i n \phi-i m \theta-i \omega t)$ with:

$$
\frac{\partial \delta \hat{\dot{f}}_{j k}}{\partial \theta}=i q\left(\omega \frac{\partial F_{j}}{\partial \mathcal{E}}-n \frac{\partial F_{j}}{\partial \mathcal{P}_{\phi}}\right) \delta \hat{U}^{\theta} \frac{\partial}{\partial \theta}\left(R v_{\|}\right)
$$

A constant of integration must be evaluated by applying boundary conditions to the drift kinetic equation at the next order on Larmor radius (or more exactly in banana width relative to the length scale of the mode in question). On calculating the pressure moments associated with $\dot{\delta f_{j k}}$, substituting these into the momentum equation of Eq. (34), and undertaking the vorticity operator $V[\boldsymbol{X}]$ of Eq. (20), one obtains the kinetically enhanced inertia. In particular, one finds [23] for $n=0$ :

$$
\begin{array}{r}
V\left[-i \rho \omega \delta \dot{\boldsymbol{U}}_{\perp}-\left(\dot{\boldsymbol{P}}_{\perp}-\dot{\delta} \dot{P}_{\|}\right) \boldsymbol{\kappa}+\nabla_{\perp} \dot{\delta} \dot{P}_{\perp}\right] \propto \\
-\rho \omega^{2} \delta U^{\theta}\left(1+1.6 q^{2} \epsilon^{-1 / 2}\right) .
\end{array}
$$

The inertial enhancement $1 \rightarrow 1+1.6 q^{2} \epsilon^{-1 / 2}$ can be compared with that of static ideal MHD $1 \rightarrow 1+2 q^{2}$ (see Eq. (23) with $\Omega=0$ and $M=0$ ) which in the ideal MHD case arises from the compressibility contribution $-\Gamma P \nabla \cdot \boldsymbol{\delta} \boldsymbol{U}$ to $\delta P$. It might be of interest to note in the context of zonal flows that the factor $1.6 q^{2} \epsilon^{-1 / 2}$ was independently obtained in Ref. [26] as a factor describing non-linear residual zonal flow assuming a given source.

Returning to the problem at hand, for the case of $\omega=$ 0 , and $n=0$, then from Eq. (45) one obtains $\dot{\delta f} f_{j k}=0$. Furthermore, with $\boldsymbol{\delta} \boldsymbol{A}=\boldsymbol{\delta} \boldsymbol{U} \times \boldsymbol{B}$, it is easily shown that $\delta A^{\phi}=\delta U^{\psi} / R^{2}$. Again, assuming that ideal MHD results hold, whereby $\delta U^{\psi}=0$ and $\boldsymbol{\delta} \boldsymbol{B}=0$, it is thus clear from Eq. (38) that the total (fluid plus kinetic) perturbed distribution function $\dot{\delta f}$ for each species is zero, and hence so are their moments:

$$
\dot{\delta} \rho_{j}=0, \quad \dot{P}_{j \perp}=0, \text { and } \quad \dot{P}_{j \|}=0
$$

As a result of this, the continuity equation of Eq. (44) simply yields $\rho_{j} \boldsymbol{\nabla} \cdot \boldsymbol{\delta} \boldsymbol{U}_{j}=0$. Since the momentum equation is indeed balanced with $\boldsymbol{\delta} \boldsymbol{B}=0$, the kinetic-MHD problem simply reverts to the ideal MHD problem already described, and the mode structure is exactly that calculated in section IIIA.

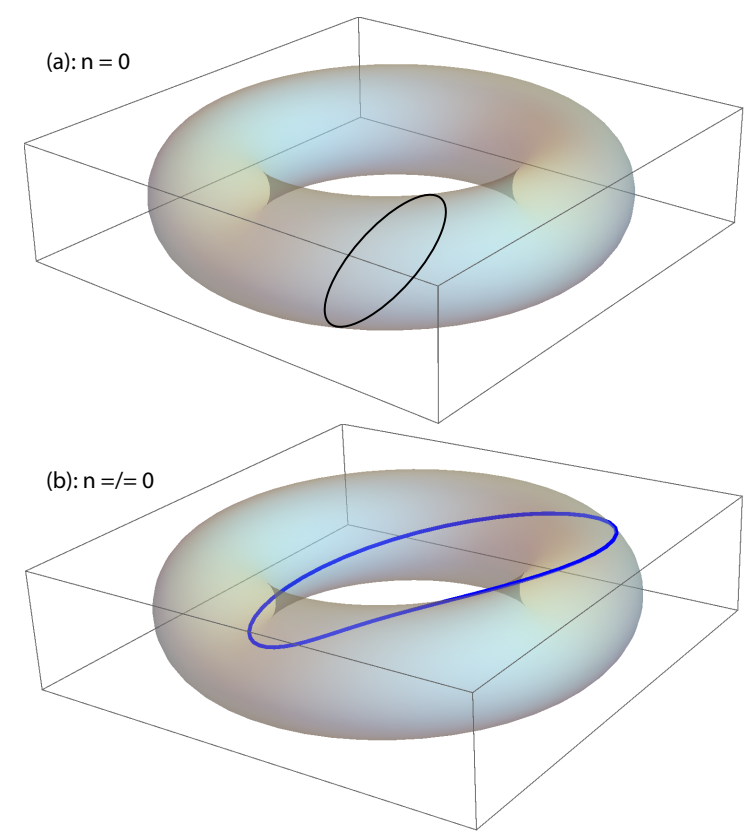

FIG. 1: Showing schematically the trajectory of $\boldsymbol{\delta} \boldsymbol{U}$ for (a) $n=0$ zonal flows, and (b) $\mathrm{n}=1$ zonal modes on the $q=1$ surface.

\section{B. Non-axisymmetric zonal modes under kinetic-MHD model}

In this section on non-axisymmetric zonal modes we again assume initially that the modes obey ideal MHD such that $\delta \phi=0, \boldsymbol{\delta} \boldsymbol{B}=0$, and for $n \neq 0, \boldsymbol{\delta} \boldsymbol{U}=\delta U_{\|} \boldsymbol{b}$, or $\delta U^{\phi}=q \delta U^{\theta}$. Thus, from Eqs. (33) and (41), $\dot{\delta A}=\left(q \delta U^{\theta}-\delta U^{\phi}\right) \nabla \psi=0$, and thus the perturbed Lagrangian $\dot{\delta L}$ is also zero.

With $\dot{\delta}=0, \delta \phi=0, \delta B_{\|}=0$ and $\delta A^{\phi}=0$, then $\delta f_{j}=0$ for all species, and so are their moments. As in the last subsection, the problem then reverts to the ideal MHD problem, and the form of the flow components follow those obtained in Section IIIB. Note that in the case of $n \neq 0$, the modes are again purely hydrodynamic.

Now that the exact zonal mode structures have been confirmed using kinetic-MHD, Fig. 1 plots schematically typical trajectories for (a) an $n=0$ zonal flow, and (b) an $n=1$ zonal mode on the $q=1$ surface. Note that in both cases, the flows close on themselves. In the case of $n \neq 0$, the flow closure is required mathematically, while for $n=0$, the flow closure has been imposed by choosing a specific value for the iso-rotation [21], as discussed in Section III.

\section{CONCLUSIONS}

This paper has attempted to provide a coherent picture of zonal modes in MHD and kineitc-MHD models in axisymmetric magnetic confined plasmas. An obvious 
starting place was to show that the MHD model yields zonal modes and GAMs as two branches of slow magnetosonic continua. This is more convincingly done in toroidally rotating equilibria, where the zonal mode has finite frequency in the rotating plasma frame, verifying that zonal modes are genuine continuum modes. The paper has shown a straightforward and clear methodology for obtaining mode continua in rotating plasma, hopefully providing a useful reference for such calculations. The study in rotating plasmas identified that nonaxisymmetric zonal modes exist, and this in turn motivated a study into the exact structure of zonal modes using the ideal MHD model. It was shown that nonaxsymmetric zonal flows are purely hydrodynamic in static plasmas, highly localised over flux label, and the flows close on themselves, identically following magnetic field lines on rational surfaces. The zonal flow closure, or return flow, is a feature shared with $n=0$ zonal flows, as also discussed in this paper.

Much is already known about $n=0$ zonal modes in static equilibria. However, probably less well known, and in part motivating some of the work contained in this paper, is that $n=0$ zonal modes do acquire a finite and potentially measurable frequency in rotating plasmas. This finite frequency could be connected with the $n=0$ modes observed on the tokamak threshold between L-mode and $\mathrm{H}$-mode [7]. One may ask about the relevance or importance of highly localised $n \neq 0$ zonal modes. Under the ideal MHD model, it is know that in plasmas with equilibrium toroidal rotation, $n \neq 0$ zonal modes rotating at the Brunt Vaisala frequency couple with pressure driven instabilities. This effect is seen by applying the voticity operator of Eq. (20) on the momentum equation for the pressure driven instability. It is then found that the inertia associated with the instability has an enhancement defined by Eq. (23). The dispersion relation for the ideal MHD instability, whose effects of toroidal rotation are almost exclusively confined to the inertial enhancement, yields complex frequencies and thus modified growth rates [27]. These physical phenomena have been verified in a detailed study of the internal kink mode [8], where analytic work has carefully identified that centrifugal and coriolis effects effects are physical, and where benchmarking against a full MHD codes in which toroidal rotation was self consistently included in the equilibrium and the linearly perturbed MHD equations.

The mechanism for coupling between $n \neq 0$ zonal modes and pressure driven instabilities is not yet known in a rotating kinetic description of the plasma. But having seen the important coupling effect in ideal MHD plasmas, we can expect crucial modifications in a more self consistent kinetic-MHD description. A significant start to this modelling has been made in the current paper where kinetic MHD has been employed to verify the results obtained for zonal modes in ideal MHD plasmas, and where ideal MHD rotating equilibria have been verified kinetically. It is hoped that these advanced kinetic studies will not only provide an interesting academic study into corrections to continua, and the structure of zonal modes, but might provide insight into kinetic damping of pressure driven instabilities, and new means to control them empirically.

\section{Acknowledgements}

This work has been partially supported by the Swiss National Science Foundation.
[1] P. H. Diamond et al, Plasma Phys. Control. Fusion 47, R35 (2005)

[2] K. Itoh, S.-I. Itoh, P. H. Diamond, T. S. Hahm, A. Fujisawa, G. R. Tynan, M. Yagi, and Y. Nagashima, Physics of Plasmas 13, 055502 (2006)

[3] K Itoh, K Hallatschek and S-I Itoh, Plasma Phys. Control. Fusion 47, 451 (2005)

[4] C. Wahlberg and J. P. Graves, Plasma Phys. Control Fusion 58, 075014 (2016)

[5] C. Wahlberg, Plasma Phys. Control. Fusion 51085006 (2009)

[6] J. P. Graves et al, Nature Communs. 3, 624 (2012)

[7] N. Vianello et al., "M-mode and HFO in JET" 42nd EPS Conference on Plasma Physics (2015), P2.133

[8] C. Wahlberg, I. T. Chapman and J. P. Graves, Phys. Plasmas 16, 112512 (2009)

[9] E. R. Priest, Solar Magnetohydrodynamics (Reidel, Dordrecht, 1982), Chap. 4.

[10] C. Wahlberg, Phys. Rev. Lett. 101, 115003 (2008)

[11] J. P. Goedbloed, Phys. Fluids 18, 1258 (1975)

[12] J. W. Havenkort, H. J de Blank and B. Koren, Plasma Phys. Cont. Fusion 53, 043004 (2011)
[13] A. J. C. Beliën et al, J. Comput. Phys. 182, 91 (2002)

[14] P. J. Catto, Phys. Fluids 30, 2784 (1987)

[15] H. Grad "The guiding center plasma" , in Proceedings of Synopsia in Applied Mathematics (American Mathematical Society), Providence, Rhode Island, 1966, Vol 18, p.162.

[16] E. Frieman and and M Rotenburg, Rev. Mod. Phys. 32, $898(1960)$

[17] J. P. Goedbloed, R. Keppens, S. Poedts, "Advanced Magnetohydrodynamis, with applications to Laboratory and Astrophysical Plasmas," Cambridge University Press, 2010

[18] M. N. Bussac, R. Pellat, D. Edery and J. L. Soulé, Phys. Rev. Lett. 35, 1638 (1975).

[19] A. B. Mikhailovskii 1986 Reviews of Plasma Physics vol 9 ed M A Leontovich (New York: Consultants Bureau)

[20] A. B. Mikhailovskii 1998 Instabilities in a Confined Plasma (Bristol: Institute of Physics)

[21] V. C. A. Ferraro, "The non-uniform rotation of the sun and its magnetic field." Notices Roy. Astr. Soc. 97, 458472 (1937)

[22] T. M. Antonsen Jr. and A Bondeson, Phys. Rev. Lett. 
71, 2046 (1993)

[23] J. P. Graves et al Plasma Phys. Cont. Fusion 40, 1049 (2000)

[24] F. Porcelli et al Phys. Plasmas 1, 470 (1994)

[25] J. P. Graves, Plasma Phys. Control. Fusion 55074009
(2013)

[26] M. N. Rosenbluth and F. L. Hinton, Phys. Rev. Lett. 80, $724(1998)$

[27] F. L. Waelbroeck, Phys. Plasmas 31047 (1996) 